\title{
Calcineurin and skeletal muscle growth
}

\author{
Robin N. Michel ${ }^{1 *}$, Shannon E. Dunn ${ }^{1}$ and Eva R. Chin ${ }^{2}$ \\ ${ }^{1}$ Neuromuscular Research Laboratory, Department of Chemistry and Biochemistry, Laurentian University, Sudbury, \\ Ontario P3E 2C6, Canada \\ ${ }^{2}$ Department of Cardiovascular and Metabolic Diseases, Pfizer Global Research \& Development, Eastern Pt Rd, \\ MS8220-3120, Groton, CT 06340, USA
}

\begin{abstract}
Recruitment determines the profile of fibre-type-specific genes expressed across the range of muscle fibres associated with slow, fast fatigue-resistant and fast fatiguable motor units. Downstream signalling pathways activated by neural signalling and mechanical load have been the focus of intensive research in past years. It is now known that $\mathrm{Ca}^{2+}$-dependent calcineurin-nuclear factor of activated T cells and insulin-like growth factor 1 pathways and their downstream mediators contribute to these adaptive responses. These pathways regulate gene expression through muscle-specific (myocyte-enhancing factor 2, myoblast determination protein) and non-specific (nuclear factor of activated T cell 2, GATA-2) transcription factors. Transcriptional signals activated with increased contractile activity result in altered expression of fibre-type specific genes, including the myosin heavy chain isoforms and oxidative and glycolytic enzymes and a net change in muscle fibre-type composition. In contrast, transcriptional signals activated by increased load bearing result in hypertrophy or a growth response, a component of which involves satellite cell recruitment and fusion with existing adult myofibres. Calcineurin has been identified as a key mediator in the hypertrophic response, and the current challenge has been to determine the downstream target genes of this pathway. Exciting new data have emerged, showing that myostatin, a negative regulator of muscle growth, and utrophin, a cytoskeletal protein important in maintaining membrane integrity, are downstream targets of calcineurin signalling. Increased understanding of these mediators of muscle growth may provide strategies for the development of effective therapeutics to counter muscle weakness and muscular dystrophy.
\end{abstract}

Skeletal muscle hypertrophy: Myostatin: Nuclear factor of activated $\mathbf{T}$ cell and myocyte-enhancing factor 2 signalling

Although the contractile and metabolic phenotype of a muscle fibre is genetically pre-determined and modelled by developmental surges in thyroid hormone and other endocrine signals, it remains highly plastic in the adult mammal and can be adjusted to match the functional demands placed on it by the central nervous system. The diversity in muscle fibre expression and accumulation of contractile proteins in adult mammals appears to be, in large part, directed by neural activity. Depolarization of the sarcolemma in response to motor neuron firing triggers a release of $\mathrm{Ca}^{2+}$ from the sarcoplasmic reticulum and subsequent contraction of the muscle fibre. The amount and pattern of nerve-mediated depolarizations have a large influence on the expression and accumulation of contractile proteins within skeletal muscle fibres. It is well known that when muscles are denervated and then activated with patterns of electrical stimuli modelled after non-native nerve discharges they will convert to a phenotype consistent with the new neural activation profile. Thus, with appropriate stimuli, slow-twitch muscles can adapt to display a faster myosin heavy chain (Mhc) profile and fasttwitch muscles can switch over to a slower Mhc phenotype (Ausoni et al. 1990; Dunn \& Michel, 1997, 1999). The fact that similar transformations in contractile proteins can be induced in cultured skeletal myotubes by modulating intracellular $\mathrm{Ca}^{2+}$ levels (Meissner et al. 2001) suggests the nerve may direct muscle-fibre phenotype via the fluctuations in $\mathrm{Ca}^{2+}$ that it evokes in these cells. Over the past few years some of the cellular and molecular factors that mediate these specific fibre responses to nerve-mediated depolarizations have been identified. Specifically, it has been shown that calcineurin $(\mathrm{Cn}), \mathrm{a} \mathrm{Ca}^{2+}$-regulated phosphatase,

\footnotetext{
Abbreviations: CaM, calmodulin; Cn, calcineurin; CsA, cyclosporine A; IGF-1, insulin-like growth factor 1; MEF2, myocyte-enhancing factor 2; Mhc, myosin heavy chain; NFAT, nuclear factor of activated T cells.

* Corresponding author: Dr Robin N. Michel, fax +1 705673 6508, email RNMichel@ nickel.laurentian.ca
} 


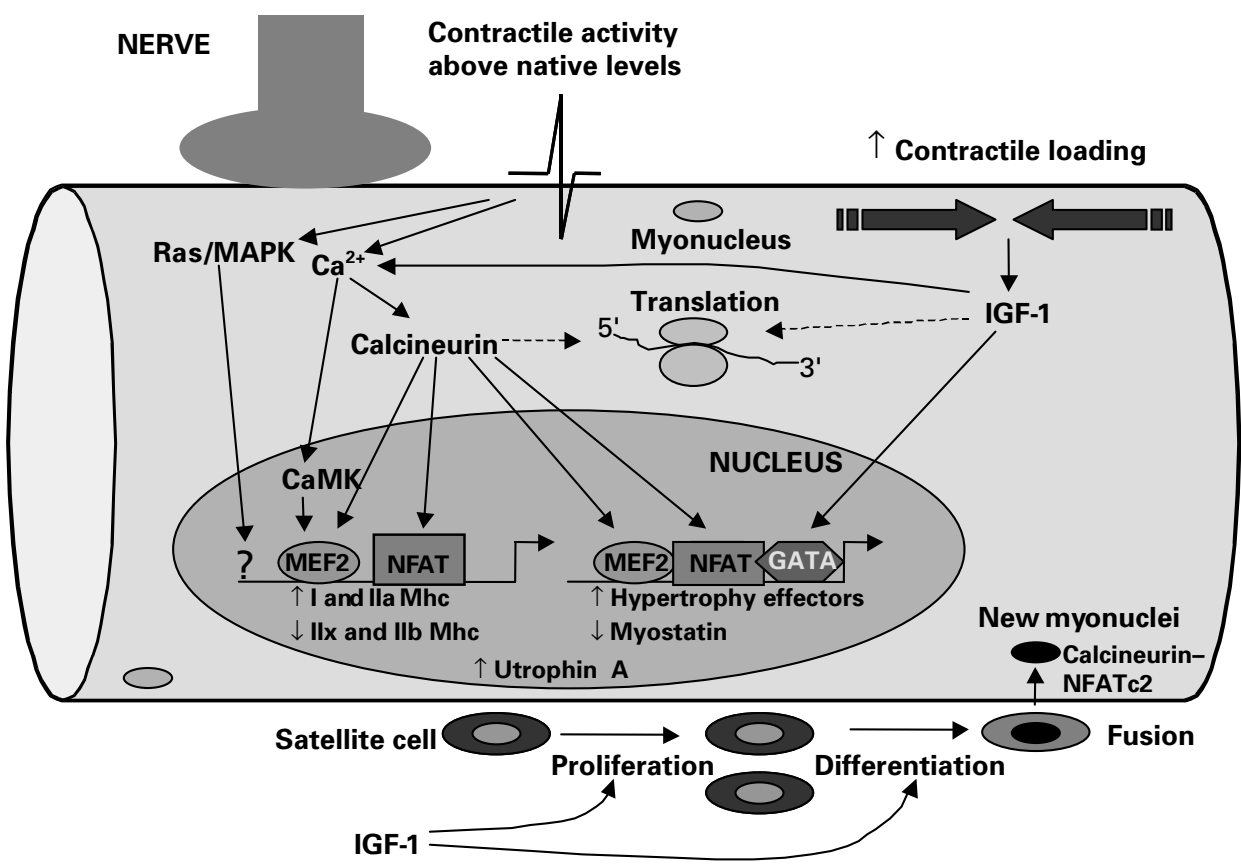

Fig. 1. Overview of muscle signalling mechanisms. Neural activation and mechanical loading of the muscle activate numerous intracellular signals that result in hypertrophic growth of the muscle. Neural recruitment results in sarcolemmal membrane depolarizations and subsequent increases in intracellular $\mathrm{Ca}^{2+}$ levels, with the resultant intracellular $\mathrm{Ca}^{2+}$ concentration dependent on the stimulation frequency. This increase in intracellular $\mathrm{Ca}^{2+}$ can activate a number of transcriptional pathways including the $\mathrm{Ca}^{2+} /$ calmodulin-dependent phosphatase calcineurin and $\mathrm{Ca}^{2+} /$ calmodulindependent kinase (CaMK) pathways. Calcineurin and its downstream transcription factor nuclear factor of activated T cells (NFAT) play an important role in the activation of type I and Ila myosin heavy chain (Mhc), oxidative enzyme and utrophin A genes as well as in the hypertrophy response through decreases in myostatin expression and altered expression of other unknown targets. Calcineurin also acts through myocyte-enhancing factor 2 (MEF2)-dependent transcription. In parallel with the calcineurin-NFAT and calcineurin-MEF2 signals, other intracellular signals are activated to effect growth. These signals are triggered by membrane depolarizations, contractile loading or the release of growth factors, such as insulin-like growth factor 1 (IGF-1), which activate hypertrophic responses through Ras/mitogen-activated protein kinase (MAPK), phosphatidylinositol 3-kinase and Akt/protein kinase B. One of the downstream effects of calcineurin and IGF-1 is activation of satellite cell proliferation and fusion with existing myofibres, which contributes, in part, to the resultant hypertrophy. $\uparrow, \downarrow$, Increase and decrease respectively; $\longleftarrow$, established pathways; $---\rightarrow$, possible pathways.

is a crucial modulator of skeletal muscle hypertrophy of all fibre types, fast or slow, in vivo (Dunn et al. 1999) and that Cn regulates fibre-type-specific genes (Chin et al. 1998; Dunn et al. 1999). In addition to the neural-evoked signals, increased muscle loading can also activate the release of various growth factors, including insulin-like growth factor 1 (IGF-1), which signals through both Cn-dependent and Cn-independent pathways (see Fig. 1) to induce muscle hypertrophy. The present paper will review both $\mathrm{Cn}-$ dependent and $\mathrm{Cn}$-independent mechanisms of skeletal muscle growth and the downstream targets of $\mathrm{Cn}$ dependent signalling.

\section{Calcineurin regulation of skeletal muscle growth}

The $\mathrm{Ca}^{2+} /$ calmodulin (CaM)-dependent phosphatase $\mathrm{Cn}$ (or protein phosphatase-2B) has recently come to the forefront as a molecular translator of low-amplitude sustained $\mathrm{Ca}^{2+}$ oscillations in skeletal muscle cells, as with other cell types (Bito et al. 1996; Dolmetsch et al. 1997; Olson \& Williams, 2000a,b). Initial data implicating $\mathrm{Cn}$ in such a role have been provided in lymphocytes in which $\mathrm{Cn}$ signals to its target transcription factor, nuclear factor of activated T cells (NFAT), in response to low-amplitude prolonged-duration intracellular $\mathrm{Ca}^{2+}$ transients, such as those that occur in response to antigenic stimulation (Dolmetsch et al. 1997). Cn-NFAT has been shown to discriminate amongst different patterns of electrical stimuli in skeletal muscle cells (Liu et al. 2001). Activation of $\mathrm{Cn}$ in response to prolonged contractile work or during muscle fibre maturation is crucial to signalling the expression of slower more oxidative fibre-specific genes (Serrano et al. 2001) and skeletal muscle growth (Dunn et al. 1999; Mitchell et al. 2002). The in vivo administration of $\mathrm{Cn}$ inhibitors, cyclosporine A (CsA) or FK506, blocks muscle fibre hypertrophy in response to functional overload (Dunn et al. 1999) and hinders the recovery of muscle fibre size after unweighting-induced atrophy (Mitchell et al. 2002). 
Additionally, administration of these $\mathrm{Cn}$ inhibitors induces slow-to-fast phenotype transformations in the normal weight-bearing or regenerating soleus (Chin et al. 1998; Bigard et al. 2000; Serrano et al. 2001) and prevents type $\mathrm{IIb} \rightarrow \mathrm{IIx} \rightarrow \mathrm{IIa} \rightarrow \mathrm{I}$ Mhc transitions in the functionallyoverloaded plantaris (Dunn et al. 1999).

Cn-dependent signalling events are required for growth to occur in all (i.e. fast and slow) fibre types. This requirement was initially shown by studies in which the $\mathrm{Cn}$ inhibitors CsA or FK506 were administered to mice or rats at a dosage $(25 \mathrm{mg} / \mathrm{kg})$ that reduces $\mathrm{Cn}$ activity in skeletal muscles by $\geq 50 \%$. This treatment prevents hypertrophy of all fibre types in the plantaris as well as fibre type transformations in response to functional overload (Dunn et al. 1999, 2000) without affecting animal health or ambulation (Dunn et al. 2002). A role for CaM-dependent signalling via $\mathrm{Cn}$ in the regulation of growth has also been identified. Overexpression of a binding peptide that inhibits $\mathrm{CaM}$ from signalling to target enzymes (CaM inhibitory peptide) mimics the effects of $\mathrm{Cn}$ inhibitors and blocks growth of overloaded muscle fibres that express this transgene (Dunn et al. 2000). This molecular-based evidence supports the pharmacological data and collectively provides convincing evidence for the role of $\mathrm{Ca}^{2+}$ and Cn-NFAT signalling in the hypertrophic response related to overload.

Controversy in the literature concerning the role of $\mathrm{Cn}$ dependent signalling pathways in adaptive growth appears to be related to the dose dependency of CsA action (Dunn et al. 2002). CsA regimens using lower dosages (i.e. $5-10 \mathrm{mg} / \mathrm{kg}$ ) and frequencies (once daily) of drug administration result in less-marked inhibition of $\mathrm{Cn}$ activity (i.e. $\leq 20 \%$; Dunn et al. 2002; Fig. 2). These lower doses of CsA do not prevent muscle fibre hypertrophy in overloaded or regenerating rat skeletal muscles (Bodine et al. 2001; Serrano et al. 2001), although they are effective in inducing fibre phenotype transformations in the rat soleus (Chin et al. 1998; Bigard et al. 2000; Serrano et al. 2001). This CsA dose dependency has recently been confirmed in overloaded muscles (SE Dunn and RN Michel, unpublished results), showing clearly that $25 \mathrm{mg} / \mathrm{kg}$ twice daily effectively blocks hypertrophy of all plantaris fibre types whereas a dose of $10 \mathrm{mg} / \mathrm{kg}$ per $\mathrm{d}$ does not (see Fig. 2(a and b)). The fact that fibre hypertrophy is not prevented when Cn activity is only modestly compromised suggests that parallel signalling pathways co-regulate growth and can compensate to induce hypertrophy under these conditions. Consistent with this notion, protein levels of the growth effector IGF-1, as well as muscle regulatory genes and transcription factor proteins targeted by IGF-1 signalling such as myogenin (Adi et al. 2000) and GATA-2 (Musaro et al. 1999, 2001), are up regulated in a compensatory fashion in overloaded muscles when $\mathrm{Cn}$ activity is compromised with CsA (Dunn et al. 2000; Fig. 3). The requirement of accessory parallel signalling pathways for growth is further supported by the finding that overexpression of constitutively-active $\mathrm{Cn}$ in $\mathrm{Tg}$ mice does not induce skeletal muscle hypertrophy in normal weightbearing animals (Dunn et al. 2000; Naya et al. 2000), nor does it potentiate fibre growth in response to overload (Dunn et al. 2000).
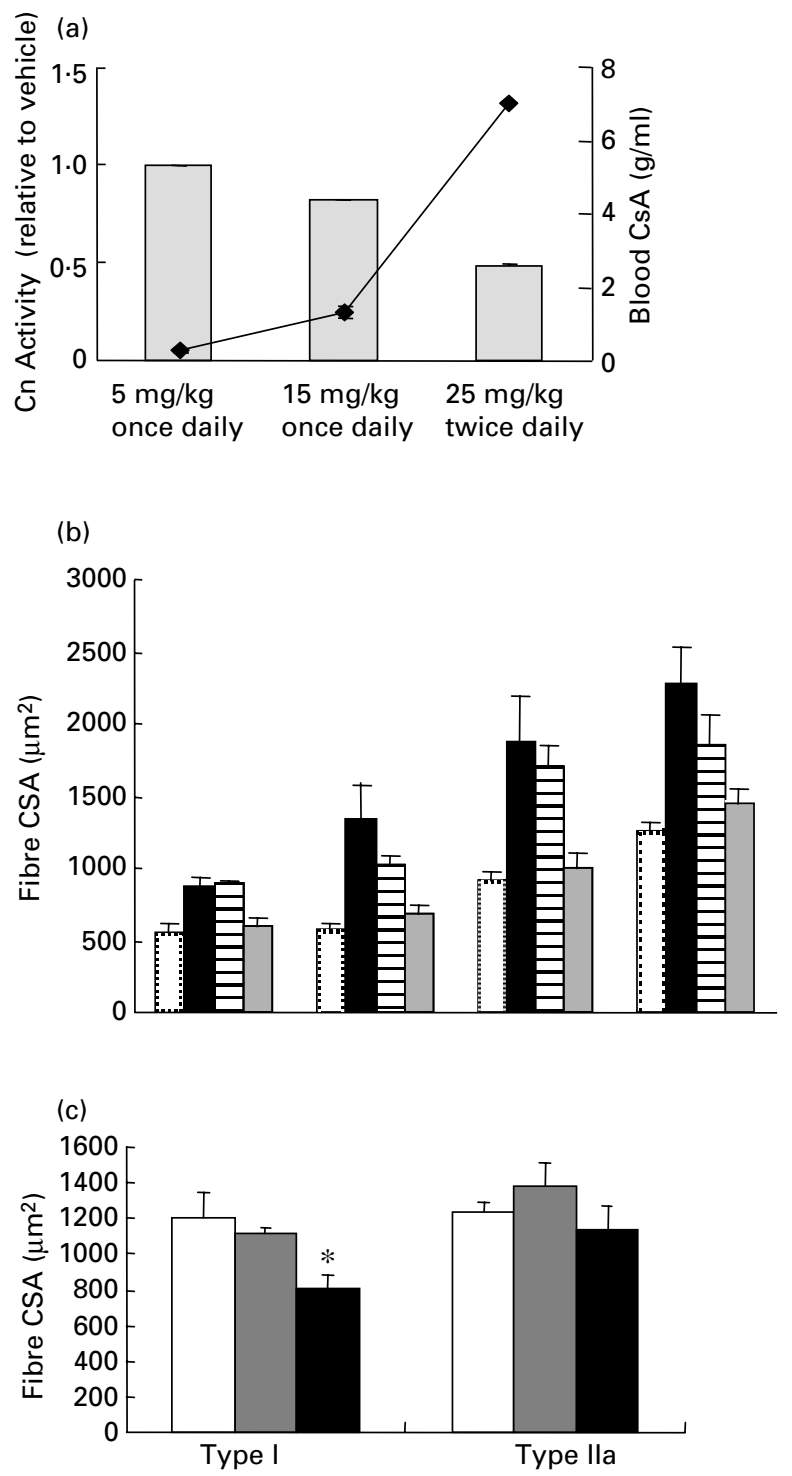

Fig. 2. Dose-dependent inhibition of compensatory overload and normal weight-bearing responses with calcineurin $(\mathrm{Cn})$ and calmodulin inhibitors. (a) CD-1 mice were administered cyclosporin $A$ (CsA) at 5 or $15 \mathrm{mg} / \mathrm{kg}$ per $\mathrm{d}$ or $25 \mathrm{mg} / \mathrm{kg}$ twice daily, resulting in dose-dependent increases in blood CsA level $(\bullet)$ and decreases in muscle Cn activity ( $\square$ ). (b) Overload hypertrophy of the plantaris muscle following removal of the soleus and gastrocnemius muscles (Ov) resulted in increased fibre cross-sectional area (CSA) of muscle fibres of all types (Ov-vehicle-treated (ם) $v$. shamoperated-vehicle-treated $(\square)$ ). This effect was inhibited by CsA treatment at $25 \mathrm{mg} / \mathrm{kg}$ twice daily $(\square)$ but not at $10 \mathrm{mg} / \mathrm{kg}$ per d (曰). (c) Regenerating muscle fibre CSA in normal weight-bearing animals was reduced in type I but not in type Ila fibres from the 5229 line of transgenic mice $(\boldsymbol{\square})$ that overexpress a calmodulin inhibitory peptide (CaMBP) driven by the troponin I slow promoter, which limits expression to type I fibres. Wild-type mice $(\square)$ and transgenic mice from the 6444 line $(\square)$, which have lower expression of the CaMBP transgene, had higher type I CSA. Values are means with their standard errors represented by vertical bars. Mean value was significantly lower than that for the other two groups: ${ }^{\star} P<0.05$. 
Cn-dependent signalling pathways may also play a role in the maintenance of fibre size in specific muscles under normal weight-bearing conditions. Administration of CsA $(25 \mathrm{mg} / \mathrm{kg}$ per d) orally to rats over 3 weeks causes plantaris and soleus muscles to lose some of their mass (Bigard et al. 2000). In contrast, administration of this same CsA dosage subcutaneously, or effective doses of FK506, does not affect the size of plantaris fibres in mice (Dunn et al. 1999, 2000). This discrepancy may relate to species-specific differences in the uptake, tissue distribution, clearance and in vivo effects of these drugs. Nonpharmacological evidence, however, suggests that $\mathrm{Ca}^{2+}$ / $\mathrm{CaM}$-dependent signalling is required for the maintenance of fibre size. There is a marked decrease in the average size of regenerating soleus slow type I fibres in $\mathrm{Tg}$ mice overexpressing $\mathrm{CaM}$ inhibitory peptide, driven by the troponin I slow promoter (Dunn et al. 2000), under normal weight-bearing conditions. This effect is also dosedependent, since a line of $\mathrm{Tg}$ mice (line 5229) with high levels of CaM inhibitory peptide expression display significantly $(P<0.05)$ smaller regenerated calibre type I fibres compared with a $\mathrm{Tg}$ line (line 6444) with lower levels of $\mathrm{CaM}$ inhibitory peptide expression (see Fig. 2(c)). These data suggest that maintenance of growth is dose-dependently related to the level of $\mathrm{Ca}^{2+} / \mathrm{CaM}$-dependent signalling through $\mathrm{Cn}$. Nonetheless, the effects of CsA and CaM inhibitory peptide on fibre size are subtle in the control or regenerating condition compared with the hypertrophic condition, emphasizing that $\mathrm{Cn}$ signalling pathways play a more substantive role in regulating fibre growth than in maintenance of size. In summary, $\mathrm{Cn}$ is necessary for skeletal muscle growth and appears to be the primary, although not the only, modulator of change in response to increased neural activation (Dunn et al. 1999, 2000, 2001).

\section{Downstream mediators of calcineurin-nuclear factor of activated $\mathbf{T}$ cell signalling}

Although $\mathrm{Cn}$ appears critical to mediating skeletal muscle hypertrophy, the downstream effector genes or targets in this process have yet to be clearly defined. Various downstream mediators of Cn-dependent signalling have been hypothesized (see Fig. 1) and include NFAT and myocyte-enhancing factor 2 (MEF2) proteins as well as GATA transcription factors. Similar to how these factors co-stimulate the transcriptional response of certain hypertrophic marker genes in the heart (Molkentin et al. 1998; Passier et al. 2000) and affect IGF-1 growth of skeletal myocytes in vitro (Musaro et al. 1999), these downstream transcription factors also appear to regulate skeletal muscle growth in vivo. GATA-2 expression is up regulated (Dunn et al. 2000) and NFAT more extensively dephosphorylated (Dunn et al. 2001) in hypertrophying skeletal muscles in vivo, suggesting that GATA-NFAT complexes may be at the nexus of neural activity-dependent and contractile load-dependent influences in skeletal muscles, via $\mathrm{Cn}$ and IGF-1 respectively. Despite the demonstrated importance of $\mathrm{Cn}-\mathrm{NFAT}$ and $\mathrm{Cn}-\mathrm{MEF} 2$ signalling events in regulating cardiac growth and the induction of the fetal programme of gene expression (i.e. atrial natriuretic factor (ANF), type I Mhc and $\alpha$-skeletal actin) in cardiac hypertrophy (Rothermel et al. 2001), the growth-effector genes targeted by these transcription factors in both cardiac and skeletal muscle have not been identified. Findings that overexpression of constitutively-active $\mathrm{Cn}$ in cardiomyocytes is associated with the activation of c-Jun $\mathrm{N}$-terminal kinase, extracellular signal-regulated kinase and protein kinase $C \alpha$ and $-\varphi$ raises the possibility that signalling of cardiac hypertrophy by $\mathrm{Cn}$ may occur through the production of an autocrine growth factor, such as IGF1 (Molkentin, 2000). However, the links to cell proliferative factors (i.e. p21, cyclin-dependent kinase 2) that increase myocyte number or to translation initiation factors (i.e. eukaryotic initiation factor $2 \mathrm{~B}$, eukaryotic initiation factor 4E-binding protein 1) that increase protein synthesis have not been clearly established.

Recent converging lines of evidence also suggest that $\mathrm{Cn}$ may be sensitive to the loading state of the muscle and may cooperate with IGF-1 signalling pathways to effect growth. We found (SE Dunn and RN Michel, unpublished results) that there is an increase in the level of IGF-1 and the muscle regulatory factors myogenin and myf-5 at the mRNA level with overload and a further compensatory increase when Cn signalling is blocked with CsA (see Fig. 3).

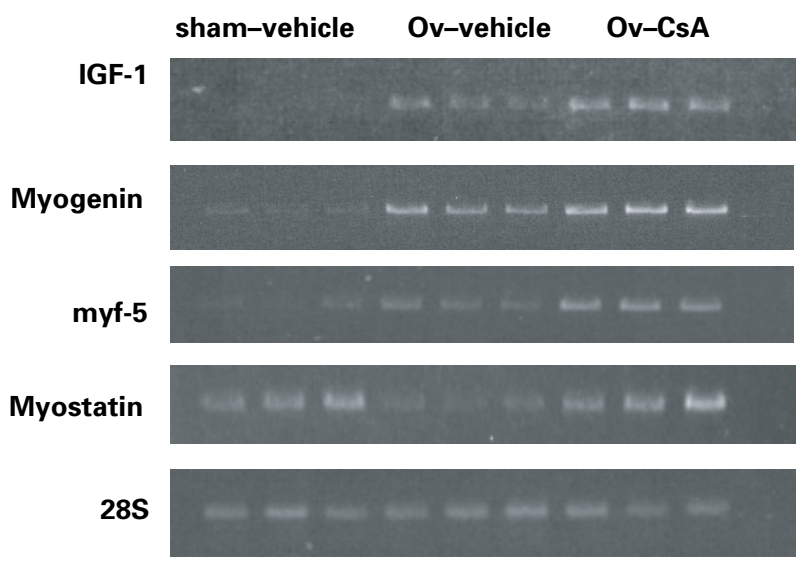

Fig. 3. Overload-induced up-regulation of IGF-1, myogenin, myf-5 and down-regulation of myostatin contributes to growth of plantaris muscle. Plantaris muscle from vehicle-treated sham-operated mice (sham-vehicle) and cyclosporin A (CsA)-treated (25 mg/kg twice daily) overloaded mice (OV-CsA) in which the soleus and gastrocnemius muscles were removed and analysed for expression of various growth-related factors. Total RNA was isolated from the distal portion of the plantaris muscle and $1 \mu \mathrm{g}$ was reversetranscribed to cDNA and amplified using primers specific for insulin-like growth factor 1 (IGF-1), the myogenic factors myogenin and myf-5, and myostatin. The $28 \mathrm{~S}$ ribosomal RNA served as a loading control. Myostatin was amplified using primers that encoded sequences that are unique to the mouse sequence of this gene (accession number 042222) and not for its closely-related counterpart GDF-11. Transcript levels of IGF-1, myogenin and myf-5 were up regulated in response to overload, and were further increased in CsA-treated mice, suggesting that the IGF-1 dependent pathway for adaptation is up regulated when calcineurin-dependent growth is blocked. Myostatin, a negative regulator of growth, was down regulated in response to overload, and this response was countered by administration of CsA. These data indicate that myostatin may be a downstream target of calcineurin-nuclear factor of activated $\mathrm{T}$ cells signalling and suggest that hypertrophic growth requires counter-regulation of growth-inhibitory pathways. 
Similarly, studies (Dunn et al. 2000) have shown an upregulation of GATA-2 with hypertrophy and a potentiated compensatory increase in this factor when Cn-mediated growth is blocked. These data strongly support parallel signalling via IGF-1-dependent and Cn-dependent pathways. Further support for a role of $\mathrm{Cn}$ signalling in response to increased load has come from studies showing $\mathrm{Cn}$ co-localization at the Z-line in cardiomyocytes, a region of the sarcomere that may play a role in the transduction of mechanical tension (Frey et al. 2000). Additionally, studies in Dictyostelium have shown that $\mathrm{Cn}$ is also activated by arachidonic acid (Kessen et al. 1999), a metabolite produced by muscles during stretch and mechanical loading (Vandenburgh et al. 1993). These data further support the notion that parallel pathways are required for adaptation in response to overload and suggest that compensatory increases in IGF-1, and possibly other signalling intermediates yet to be identified, signal via myogenin and myf-5 to increase myofibre growth. The IGF-1 pathways appear to be up regulated in a compensatory fashion when $\mathrm{Cn}$-dependent signalling is inhibited.

\section{Role of satellite cells in calcineurin-dependent and calcineurin-independent hypertrophy}

Skeletal muscle fibre hypertrophy is characterized by an expansion of the size and number of myofibrils (Rosenblatt \& Woods, 1992). This anabolic process is mediated by increases in muscle fibre transcriptional capacity and protein synthesis (Carson, 1997), and the activity-dependent regulated assembly of newly-translated proteins into sarcomeres (De Deyne, 2000; Torgan \& Daniels, 2001). Increases in muscle fibre transcriptional capacity occur, in part, through the addition of satellite cell nuclei to growing fibres (Carson, 1997). Growth factors such as IGF-1, which are released from muscle fibres in response to increased contractile loading, cause satellite cells to proliferate, differentiate and fuse with pre-existing fibres, thus maintaining nucleus:cytoplasm of the fibre during growth (Adams \& McCue, 1998). Recent findings suggest loadinduced increases in protein synthesis are mediated in large part by the Akt-mammalian target of rapamycin pathway, in which mammalian target of rapamycin phosphorylates factors (i.e. $70 \mathrm{kDa}$ ribosomal $\mathrm{S} 6$ protein kinase and eukaryotic initiation factor 4E-binding protein 1) involved in regulating the initiation factors of protein translation. Interference in either mammalian target of rapamycin signalling with rapamycin (Dunn et al. 1999; Bodine et al. 2001) or satellite cell activation via $\gamma$-irradiation of skeletal muscles (Rosenblatt \& Parry, 1993; Phelan \& Gonyea, 1997) severely hinders functional overloadinduced hypertrophy, suggesting that both processes are critical to muscle growth.

While the downstream hypertrophic factors are not well understood, it is known that $\mathrm{Cn}$ is crucial to signalling at the onset of myogenesis (Abbott et al. 1998; Musaro et al. 1999; Semsarian et al. 1999; Delling et al. 2000; Friday et al. 2000). Thus, it is possible that Cn may affect muscle fibre hypertrophy via its stimulatory effects on satellite cell differentiation. Indeed, inhibition of $\mathrm{Cn}$ activity by $\mathrm{CsA}$ or the $\mathrm{Cn}$-inhibitory peptide CAIN in cultured satellite cell lines or in primary cell culture before or coincident with growth factor withdrawal prevents their differentiation (Abbott et al. 1998; Musaro et al. 1999; Delling et al. 2000; Friday et al. 2000), whereas overexpression of constitutively-active $\mathrm{Cn}$ has the opposite effect and enhances this process (Delling et al. 2000; Friday et al. 2000). Cn may also regulate satellite cell fusion during muscle fibre hypertrophy via the NFATc2 transcription factor (Horsley et al. 2001). Primary cultures of myoblasts derived from NFATc2 $2^{-I-}$ mice, although displaying normal proliferation, differentiation and initial fusion into myotubes, fail to undergo subsequent fusion into larger more mature myotubes. This growth deficit is consistent with the phenotype of NFATc2 $2^{-I-}$ mice, in which muscle fibres are half the size and contain half the nuclei of their NFATc $2^{+/+}$counterparts.

Whether satellite cell differentiation and fusion events during mature muscle fibre growth in vivo are Cn-NFATdependent and whether the role of $\mathrm{Cn}$ in regulating muscle growth is related to the activation of satellite cells has been tested. Using a compensatory overload model, as previously described (Dunn et al. 1999, 2000), we determined that the number of new fibres at 5 and $14 \mathrm{~d}$ of compensatory overload, as determined by bromodeoxyuridine incorporation and embryonic Mhc staining, is not altered by CsA treatment despite the fact that growth is blocked in these treated muscles (see Fig. 4). Thus, while satellite cell activation may contribute to the growth of fibres during overload, this pathway does not appear to be $\mathrm{Cn}$ dependent. Furthermore, although activation of satellite cells may well be important to such muscle adaptations during overload, $\mathrm{Cn}$ appears to affect fibre growth primarily by targeting processes that are intrinsic to existing adult myofibres (Dunn et al. 2001).

\section{Insulin-like growth factor 1-induced hypertrophy: calcineurin-dependent and calcineurin-independent mechanisms}

IGF-1 is a potent anabolic agent that can activate myocyte proliferation and myocyte differentiation, leading to muscle hypertrophy. These actions occur through different signalling pathways that include the Ras/Raf-1 pathway and mitogen-activated protein kinase pathways (proliferation), the phosphatidylinositol 3-kinase-Akt-mammalian target of rapamycin pathway (protein translation) and $\mathrm{Ca}^{2+}$ / Cn-dependent pathways (differentiation and cellular hypertrophy). Overexpression of IGF-1 induces myocyte hypertrophy of both C2C12 and L6 cells in vitro (Musaro et al. 1999; Semsarian et al. 1999) and transgenic mice in vivo (Musaro et al. 2001). The muscle hypertrophy observed in vivo is accompanied by an increase in tetanic force and is the result of an increase in myofibre diameter. Interestingly, mice with muscle-specific overexpression of IGF-1 show an attenuation of the age-related decrease in muscle size and regenerative capacity in response to injury; the latter is a result of an enhanced proliferative capacity (Musaro et al. 2001). IGF-1-induced hypertrophy in vitro is also the result of increased myocyte proliferation (i.e. increased myonuclei per myotube) as well as an increased rate of protein synthesis (Semsarian et al. 1999). 
(a)

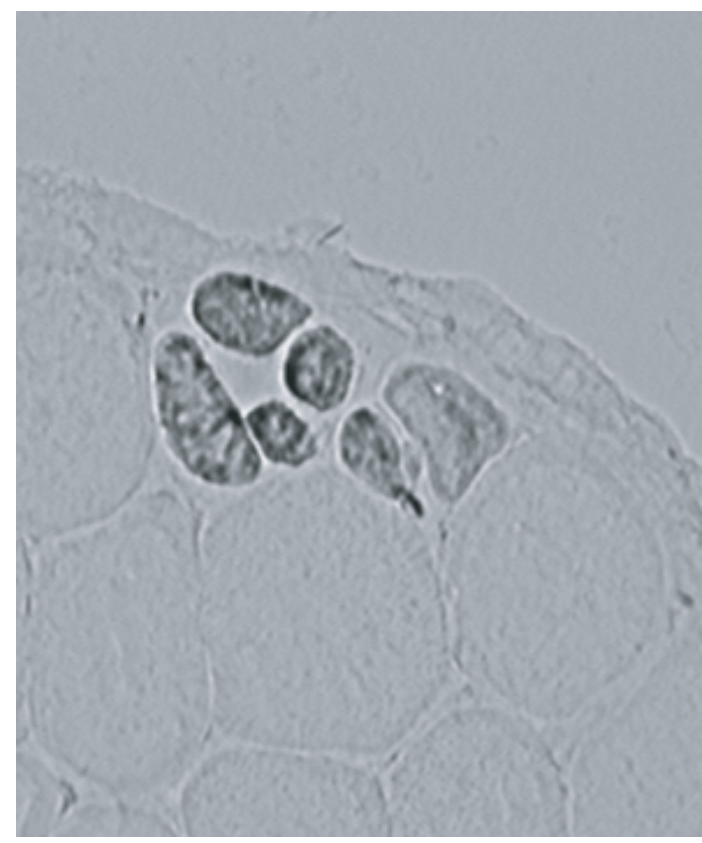

(b)

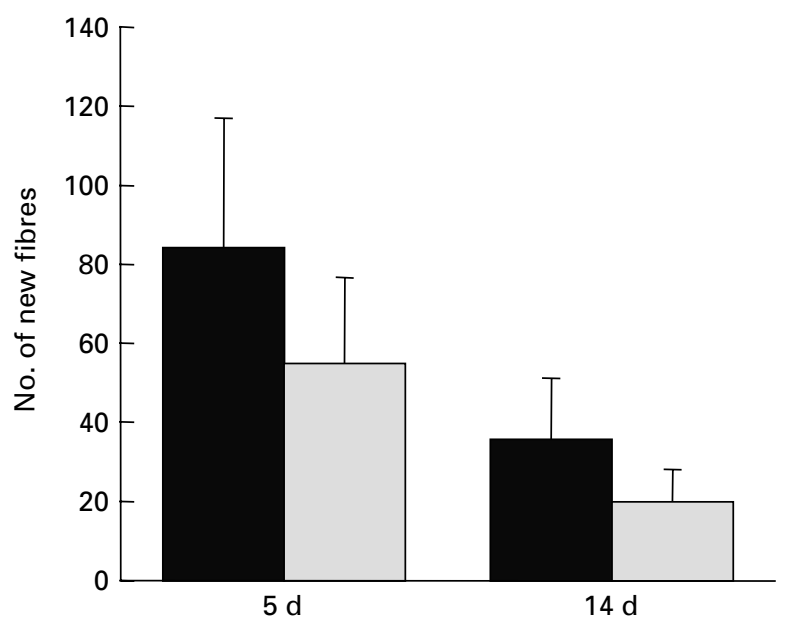

Fig. 4. Cyclosporin-dependent inhibition of muscle growth is not a result of inhibition of satellite cell differentiation. (a) Cross-sections of overloaded plantaris muscle from mice were stained for bromodeoxyuridine, a hallmark of satellite cell proliferation and fusion, or embryonic myosin heavy chain (Mhc), a marker of nascent muscle fibres. Embryonic Mhc-positive fibres are evident in the upper part of the fibre section. (b) The number of embryonic Mhc-positive fibres per muscle midbelly section were assessed in muscle from overloaded vehicle-treated ( $\mathbf{( 1 )}$ and overloaded CsAtreated $(25 \mathrm{mg} / \mathrm{kg}$ twice daily; $\square)$ mice. Values are means with their standard errors represented by vertical bars. The number of new muscle fibres per midsection at 5 and $14 \mathrm{~d}$ of overload was not affected by CsA treatment, indicating that the prevention of overload hypertrophy by calcineurin inhibitors is not the result of a blocking of satellite cell activation leading to fibre hyperplasia.

At least part of the response to IGF-1 is a result of activation of the Cn-NFAT pathway (Musaro et al. 1999; Semsarian et al. 1999) via actions of IGF-1 on L-type $\mathrm{Ca}^{2+}$ channels, resulting in an increase in intracellular free
$\mathrm{Ca}^{2+}$ concentration. Thus, increased IGF-1 would result in further increases in intracellular $\mathrm{Ca}^{2+}$ and activation of multiple $\mathrm{Ca}^{2+}$-dependent signalling pathways. Inhibition of Cn-NFAT signalling with CsA blocks the IGF-1-induced increase in $\mathrm{C} 2 \mathrm{C} 12$ proliferation, implicating this pathway in the regulation of the myocyte cell cycle. Cn-NFAT signalling also enhances myocyte fusion and thus differentiation into the more adult-like myotube state in vitro (Friday et al. 2000). Cn-NFAT signalling does not, however, appear to influence the size of myotubes once they have formed in vitro, since IGF-1 added after differentiation does not induce hypertrophy of fused C2C12 cells (Semsarian et al. 1999). This response may be different from the events that occur in vivo, since Cn-NFAT-mediated myofibre growth during muscle reloading is attenuated but not ablated in the absence of new myonuclei from satellite cells (Rosenblatt \& Parry, 1993; Mitchell \& Pavlath, 2001). Additionally, the in vivo response may involve counter-regulation of growth inhibitory pathways that may not be regulated in the same manner in vitro.

\section{Novel downstream targets of calcineurin signalling:} role of utrophin and myostatin in muscle adaptation

Activation of Cn-dependent signalling pathways is required for skeletal muscle hypertrophy (Dunn et al. 1999); however, the hypertrophic effector targets of this phosphatase have not yet been fully elucidated. Recent evidence identifies utrophin A and myostatin as possible downstream gene targets of $\mathrm{Cn}$ signalling in skeletal muscle. Utrophin is a cytoskeletal protein that forms part of the dystrosarcoglycan complex that plays an important role in maintaining membrane integrity, especially in the face of mechanical stress placed on the muscle during contractile activity. The lack of the utrophin homologue dystrophin results in muscular dystrophy and there is, therefore, therapeutic interest in the ability to up regulate utrophin expression. We have recently shown, in collaboration with Dr B Jasmin, that utrophin A is up regulated in a Cn-dependent manner, and that this gene is regulated by Cn-NFAT signalling in proportion to fibre oxidative capacity (Chakkalakal et al. 2003). Up-regulation of utrophin A in proportion to the level of fibre recruitment (i.e. type I $\geq$ IIa $>$ IIx $>$ IIb) may reflect an adaptation that allows the muscle to maintain membrane integrity in the face of increased mechanical strain related to higher levels of contractile activity.

Evidence is also reported here for the first time that $\mathrm{Cn}$ dependent down-regulation of the growth inhibitory factor myostatin occurs during overload hypertrophy and implicates myostatin as a downstream effector of $\mathrm{Cn}$-NFAT signalling (RN Michel and SE Dunn, unpublished results). Myostatin has recently come to the forefront as a negative regulator of muscle mass (McPherron et al. 1997). Mice null for myostatin display double the mass of their wildtype counterparts, which results from both increases in muscle fibre number and fibre size (McPherron et al. 1997). Moreover, overexpression of a dominant-negative form of this protein under the control of muscle creatine kinase promoter, which is expressed only in fully-differentiated 
muscle cells, leads to fibre hypertrophy (by $35 \%$ ), but does not affect fibre number (Zhu et al. 2000) This finding suggests that the effects of myostatin occur very early in development. This protein may play a role in modulating mass in response to changes in contractile loading, since its mRNA and protein levels are increased in the soleus after $10 \mathrm{~d}$ of unweighting, and this response is counteracted when muscles are periodically loaded (Wehling et al. 2000). If indeed the expression of myostatin is sensitive to loading, it would be expected that its expression would decrease in muscles when contractile loading is increased.

To test this hypothesis we investigated whether the expression of this growth factor is down regulated in mouse plantaris muscles subjected to functional overload. Moreover, to determine whether the expression of myostatin is regulated by $\mathrm{Cn}$, the sensitivity of myostatin expression to CsA treatment was examined. The plantaris in each hindlimb of CD-1 mice was overloaded via surgical removal of the gastrocnemius and soleus, and then these mice and sham controls were injected with CsA $(25 \mathrm{mg} / \mathrm{kg})$ or FK506 $(3 \mathrm{mg} / \mathrm{kg})$ or vehicle twice daily for $5 \mathrm{~d}$. mRNA levels of myostatin in these tissues were measured using RT-PCR (Dunn et al. 1999), with primers that encoded sequences that are unique to the mouse sequence of this gene but not for its closely-related counterpart, GDF-11. The transcript levels for myostatin decrease after overload and this response is countered by administration of CsA (see Fig. 3). These exciting new data suggest that activation of $\mathrm{Cn}$ in response to functional overload leads to down-regulation of the myostatin gene. This response may be of therapeutic value, since myostatin inhibition has been shown to play an important role not only in increasing muscle size but also in the prevention of obesity and type 2 diabetes (McPherron \& Lee, 2002). Thus, Cn-mediated signalling may improve the muscle mass and counter muscle weakness and various myopathies, and, more importantly, may improve overall health in individuals with these metabolic diseases. Whether the improved metabolic state with myostatin inhibition is a direct effect of the abrogation of myostatin signalling or an indirect effect of increased muscle mass, and thus increased metabolically-active tissue, is not known. It is interesting to speculate that the $\mathrm{Cn}$-dependent and myostatin signalling pathways may be counter-regulated during periods of growth and atrophy, and that therapeutic intervention of either one or both pathways would be beneficial for various metabolic disorders.

\section{Calcineurin and the acquisition of a slower more-oxidative fibre phenotype}

In addition to the role of $\mathrm{Cn}$ signalling in muscle hypertrophy, this phosphatase is known to play an important role in muscle fibre-type determination (Chin et al. 1998; Naya et al. 2000). Evidence to support this role was first provided by Chin et al. (1998), who have shown that increasing $\mathrm{Cn}$ activity in skeletal myocytes using a constitutively-active form of $\mathrm{Cn}$ induces the expression of troponin I slow and myoglobin promoters, but not the fastmuscle creatine kinase promoter. A role for $\mathrm{Cn}$ signalling in vivo has been confirmed by showing that administration of the $\mathrm{Cn}$ inhibitor $\mathrm{CsA}$ to rats induces type $\mathrm{I} \rightarrow \mathrm{II}$ fibre conversions in the soleus. The results of this study and a subsequent investigation ( $\mathrm{Wu}$ et al. 2000) have provided the framework for the original model describing the Cn-dependent control of muscle fibre phenotype. According to this model, the sustained elevations in $\mathrm{Ca}^{2+}$ that occur in muscle fibres in response to the tonic firing of slow motor nerves activates $\mathrm{Cn}$, which leads to the dephosphorylation and activation of MEF2 and NFAT proteins and the resultant transcription of slower-fibre-specific or oxidative genes (Chin et al. 1998; Wu et al. 2000). These elevations in $\mathrm{Ca}^{2+}$ also activate CaM kinases that coordinate with $\mathrm{Cn}$ to fully activate MEF2 (Wu et al. 2000). This model proposes that slow genes are selectively responsive to $\mathrm{Cn}$ signalling, because their transcriptional control regions contain NFAT and high-affinity MEF2-binding sites that are not present in the promoter regions of fast gene counterparts (i.e. troponin I fast; Chin et al. 1998; Wu et al. 2000).

The involvement of $\mathrm{Cn}$ in the signalling of a slow phenotype has since been corroborated by numerous in vitro (Delling et al. 2000; Swoap et al. 2000; Meissner et al. 2001; Torgan \& Daniels, 2001) and in vivo studies (Bigard et al. 2000; Delling et al. 2000; Naya et al. 2000; Serrano et al. 2001). However, this hypothesis has been challenged by studies reporting the induction of fast Mhc (Torgan \& Daniels, 2001) and certain fast- and musclespecific promoters (Swoap et al. 2000; Allen et al. 2001) by activated $\mathrm{Cn}$. Additionally, administration of CsA to mice, at a dose sufficient to inhibit $\mathrm{Cn}$ activity, not only blocks the accumulation of type I Mhc, but also prevents type IIb $\rightarrow$ IIx $\rightarrow$ IIa Mhc transitions in functionally-overloaded muscles (Dunn et al. 1999). Moreover, when morepotent $\mathrm{Cn}$ inhibitors (i.e. FK506 or CAIN) than CsA are used in rats, types I and IIa Mhc mRNA are both down regulated, and types IIx and IIb mRNA up regulated in regenerating and normal weight-bearing soleus muscles (Serrano et al. 2001). Further evidence supporting a role for $\mathrm{Cn}$ in mediating the expression of IIa Mhc is that stimulatory effects of the constitutively-active $\mathrm{CnA}$ transgene are much greater for IIa Mhc promoters (50- to 100fold) than for IIx or IIb Mhc promoters (5- to 10-fold; Allen et al. 2001). Additionally, myoglobin, one of the first identified gene targets of Cn (Chin et al. 1998), is expressed in highly-oxidative type IIa fibres. Thus, Cndependent control of muscle fibre phenotype appears to be directed towards genes most expressed in highly-oxidative fibres and may involve coordinated regulation of genes related to the oxidative profile of a fibre. Interestingly, $\mathrm{Cn}$ has been shown to act synergistically with $\mathrm{CaM}$ kinase IV (although not normally expressed in muscle) to activate mitochondrial biogenesis and adoption of a slower-oxidative phenotype (Wu et al. 2002). The signals downstream of CaM kinases (primarily the transcriptional cofactor PPAR $\gamma$ co-activator $1 \alpha$ ) appear sufficient to induce expression of genes related to oxidative metabolism and slower (types I and IIa) contractile elements (Lin et al. 2002; Wu et al. 2002). Thus, Cn-dependent control of fibre type may require coordination with other contractile activity-related or $\mathrm{Ca}^{2+}$-related events or signalling pathways (i.e. CaM kinases) to induce the transcription of 
myofibrillar genes co-expressed in slower, more oxidative, fibres (types I or IIa fibre-associated). These signals are transduced via either MEF2 or NFAT, but only when muscle activity is above normal or at the upper range of that associated with relatively slower more-fatigue-resistant motor unit recruitment levels. As such, $\mathrm{Cn}$ signalling appears more a vehicle for adaptation towards a moremetabolically-efficient phenotype in response to increased muscle usage than a signalling agent for exclusive maintenance of slow type I fibre profiles in response to 'slow/chronic' patterns of nerve activation.

\section{Summary}

In summary, Cn-dependent pathways that signal through NFAT- and MEF2-dependent transcriptional intermediates play an important role in myofibre growth and adaptation to increased contractile activity. This adaptation may be achieved, in part, through regulation of specific downstream targets such as utrophin A and myostatin, and is supported by the parallel activation of $\mathrm{Cn}$-independent IGF-1 and other $\mathrm{Ca}^{2+}$-dependent pathways.

\section{Acknowledgements}

We thank R. Prudhomme, A. Simard, B. J. Jasmin and R. S. Williams for their valuable scientific and technical contributions. This work was supported by grants from the Natural Sciences and Engineering Research Council of Canada and the Canadian Institutes of Health Research (to R.N.M.)

\section{References}

Abbott KL, Friday BB, Thaloor D, Murphy TJ \& Pavlath GK (1998) Activation and cellular localization of the cyclosporine A-sensitive transcription factor NF-AT in skeletal muscle cells. Molecular Biology of the Cell 9, 2905-2916.

Adams GR \& McCue SA (1998) Localized infusion of IGF-1 results in skeletal muscle hypertrophy in rats. Journal of Applied Physiology 84, 1716-1722.

Adi S, Cheng ZO, Zhang PL, Wu NY, Mellon SH \& Rosenthal SM (2000) Opposing early inhibitory and late stimulatory effects of insulin-like growth factor-I on myogenin gene transcription. Journal of Cellular Biochemistry 78, 617-626.

Allen DL, Sartorius CA, Sycuro LK \& Leinwand LA (2001) Different pathways regulate expression of the skeletal myosin heavy chain genes. Journal of Biological Chemistry 276, 43524-43533.

Ausoni S, Gorza L, Schiaffino S, Gundersen K \& Lomo T (1990) Expression of myosin heavy chain isoforms in stimulated fast and slow rat muscles. Journal of Neuroscience 10, 153-160.

Bigard X, Sanchez H, Zoll J, Mateo P, Rousseau V, Veksler V \& Ventura-Clapier R (2000) Calcineurin co-regulates contractile and metabolic components of slow muscle phenotype. Journal of Biological Chemistry 275, 19653-19660.

Bito H, Deisseroth K \& Tsien RW (1996) CREB phosphorylation and dephosphorylation: a $\mathrm{CA}^{2+}$ - and stimulus durationdependent switch for hippocampal gene expression. Cell 87, 1203-1214.

Bodine SC, Stitt TN, Gonzalez M, Kline WO, Stover GL, Bauerlein R, Zlotchenko E, Scrimgeour A, Lawrence JC, Glass
DJ \& Yancopoulos GD (2001) Akt/mTOR pathway is a crucial regulator of skeletal muscle hypertrophy and can prevent muscle atrophy in vivo. Nature Cell Biology 3, 1014-1019.

Carson JA (1997) The regulation of gene expression in hypertrophying skeletal muscle. Exercise and Sport Sciences Reviews 25, 301-320.

Chakkalakal JV, Stocksley MA, Harrison MA, Angus LM, Deschenes-Furry J, St Pierre S, Megheny LA, Chin ER, Michel RN \& Jasmin BJ (2003) Expression of utrophin A mRNA correlates with the oxidative capacity of skeletal muscle fibre types and is regulated by calcineurin. Proceedings of the National Academy of Sciences USA 100, 7791-7796.

Chin ER, Olson EN, Richardson JA, Yang Q, Humphries C, Shelton JM, Wu H, Zhu W, Bassel-Duby R \& Williams RS (1998) A calcineurin-dependent pathway controls skeletal muscle fibre type. Genes and Development 12, 2499-2509.

De Deyne PG (2000) Formation of sarcomeres in developing myotubes: role of mechanical stretch and contractile activation. American Journal of Physiology 279, C1801-C1811.

Delling U, Tureckova J, Lim HW, DeWindt LJ, Rotwein P \& Molkentin JD (2000) A calcineurin-dependent pathway regulates skeletal muscle differentiation and slow myosin heavy-chain expression. Molecular and Cellular Biology 20, 6600-6610.

Dolmetsch RE, Lewis RS, Goodnow CC \& Healy JI (1997) Differential activation of transcription factors induced by $\mathrm{Ca}^{2+}$ response amplitude and duration. Nature 386, 855-858.

Dunn SE, Burns JL \& Michel RN (1999) Calcineurin is required for skeletal muscle hypertrophy. Journal of Biological Chemistry 274, 21908-21912.

Dunn SE, Chin ER \& Michel RN (2000) Matching of calcineurin activity to upstream effectors is critical for skeletal muscle growth. Journal of Cell Biology 151, 663-672.

Dunn SE \& Michel RN (1997) Coordinated expression of myosin heavy chain isoforms and metabolic enzymes within overloaded rat muscle fibers. American Journal of Physiology 273, C371-C383.

Dunn SE \& Michel RN (1999) Differential sensitivity to myosinheavy-chain-typed fibers to distinct aggregates of nervemediated activation. Pflugers Archiv 437, 432-440.

Dunn SE, Simard AR, Bassel-Duby R, Williams RS \& Michel RN (2001) Nerve activity-dependent modulation of calcineurin signaling in adult fast and slow skeletal muscle fibers. Journal of Biological Chemistry 276, 45243-45254.

Dunn SE, Simard AR, Prud'homme R \& Michel RN (2002) Calcineurin and skeletal muscle growth. Nature Cell Biology 4, E46.

Frey N, Richardson JA \& Olson EN (2000) Calsarcins, a novel family of sarcomeric calcineurin-binding proteins. Proceedings of the National Academy of Sciences USA 97, 14632-14637.

Friday BB, Horsley V \& Pavlath GK (2000) Calcineurin activity is required for the initiation of skeletal muscle differentiation. Journal of Cell Biology 149, 657-665.

Horsley V, Friday BB, Matteson S, Kegley KM, Gephart J \& Pavlath GK (2001) Regulation of the growth of multinucleated muscle cells by an NFATc2-dependent pathway. Journal of Cell Biology 153, 329-338.

Kessen U, Schaloske R, Aichem A \& Mutzel R (1999) $\mathrm{Ca}^{2+} /$ calmodulin-independent activation of calcineurin from Dictyostelium by unsaturated long chain fatty acids. Journal of Biological Chemistry 274, 37821-37826.

Lin J, Wu H, Tarr PT, Zhang CY, Wu Z, Boss O, Michael LF, Puigserver P, Isotani E, Olson EN, Lowell BB, Bassel-Duby R \& Spiegelman BM (2002) Transcriptional co-activator PGC-1 
alpha drives the formation of slow-twitch muscle fibers. Nature 418, 797-801.

Liu Y, Cseresnyes Z, Randall WR \& Schneider MF (2001) Activity-dependent nuclear translocation and intranuclear distribution of NFATc in adult skeletal muscle fibers. Journal of Cell Biology 155, 27-39.

McPherron AC, Lawler AM \& Lee SJ (1997) Regulation of skeletal muscle mass in mice by a new TGF-beta superfamily member. Nature 387, 83-90.

McPherron AC \& Lee SJ (2002) Suppression of body fat accumulation in myostatin-deficient mice. Journal of Clinical Investigation 109, 595-601.

Meissner JD, Gros G, Scheibe RJ, Scholz M \& Kubis HP (2001) Calcineurin regulates slow myosin, but not fast or metabolic enzymes, during fast-to-slow transformation in rabbit skeletal muscle. Journal of Physiology (London) 533, 215-226.

Mitchell PO, Mills ST \& Pavlath GK (2002) Calcineurin differentially regulates maintenance and growth of phenotypically distinct muscles. American Journal of Physiology 282, C984-C992.

Mitchell PO \& Pavlath GK (2001) A muscle precursor celldependent pathway contributes to muscle growth after atrophy. American Journal of Physiology 281, C1706-C1715.

Molkentin JD (2000) Calcineurin and beyond: Cardiac hypertrophic signaling. Circulation Research 87, 731-738.

Molkentin JD, Lu JR, Antos C, Markham B, Richardson J, Robbins J, Grant SR \& Olson EN (1998) A calcineurindependent transcriptional pathway for cardiac hypertrophy. Cell 93, 215-228.

Musaro A, McCullagh KJA, Naya FJ, Olson EN \& Rosenthal N (1999) IGF-1 induces skeletal myocyte hypertrophy through calcineurin in association with GATA-2 and NFATc1. Nature 400, 581-585.

Musaro A, McCullagh K, Paul A, Houghton L, Dobrowolny G, Molinaro M, Barton ER, Sweeney HL \& Rosenthal N (2001) Localized IGF-1 expression sustains hypertrophy and regeneration in senescent skeletal muscle. Nature Genetics 27, 195-200.

Naya F, Mercer B, Shelton J, Richardson JA, Williams RS \& Olson EN (2000) Stimulation of slow skeletal muscle fiber gene expression by calcineurin in vivo. Journal of Biological Chemistry 275, 4545-4548.

Olson EN \& Williams RS (2000a) Remodeling muscles with calcineurin. Bioessays 22, 510-519.

Olson EN \& Williams RS (2000b) Remodeling muscles with calcineurin: erratum. Bioessays 22, 1049.

Passier R, Zeng H, Frey N, Naya FJ, Nicol RL, McKinsey TA, Overbeek P, Richardson JA, Grant SR \& Olson EN (2000) $\mathrm{CaM}$ kinase signaling induces cardiac hypertrophy and activates the MEF2 transcription factor in vivo. Journal of Clinical Investigation 105, 1395-1406.
Phelan JN \& Gonyea WJ (1997) Effect of radiation on satellite cell activity and protein expression in overloaded mammalian skeletal muscle. Anatomical Record 247, 179-188.

Rosenblatt JD \& Parry DJ (1993) Gamma irradiation prevents compensatory hypertrophy of overloaded mouse extensor digitorum longus muscle. Journal of Applied Physiology 73, 2538-2543.

Rosenblatt JD \& Woods RI (1992) Hypertrophy of rat extensor digitorum longus muscle injected with bupivacaine. A sequential histochemical, immunohistochemical, histological and morphometric study. Journal of Anatomy 191, 11-27.

Rothermel BA, McKinsey TA, Vega RB, Nicol RL, Mammen P, Yang J, Antos CL, Shelton JM, Bassel-Duby R, Olson EN \& Williams RS (2001) Myocyte-enriched calcineurin-interacting protein, MCIP1, inhibits cardiac hypertrophy in vivo. Proceedings of the National Academy of Sciences USA 98, 3328-3333.

Semsarian C, Wu MJ, Ju YK, Marciniec T, Yeoh T, Allen DG, Harvey RP \& Graham RM (1999) Skeletal muscle hypertrophy is mediated by a $\mathrm{Ca}^{2+}$-dependent calcineurin signaling pathway. Nature 400, 576-580.

Serrano AL, Murgia M, Pallafacchina G, Calabria E, Coniglio P, Lomo T \& Schiaffino S (2001) Calcineurin controls nerve activity-dependent specification of slow skeletal muscle fibers but not muscle growth. Proceedings of the National Academy of Sciences USA 98, 13108-13113.

Swoap SJ, Hunter RB, Stevenson EJ, Felton HM, Kansagra NV, Lang JM, Esser KA \& Kandarian SC (2000) The calcineurinNFAT pathway and muscle fiber-type gene expression. American Journal of Physiology 279, C915-C924.

Torgan CE \& Daniels MP (2001) Regulation of myosin heavy chain expression during rat skeletal muscle development in vitro. Molecular Biology of the Cell 12, 1499-1508.

Vandenburgh HH, Shansky J, Karlisch P \& Solerssi RL (1993) Mechanical stimulation of skeletal muscle generates lipidrelated second messengers by phospholipase activation. Journal of Cellular Physiology 155, 63-71.

Wehling M, Cai B \& Tidball JG (2000) Modulation of myostatin expression during modified muscle use. FASEB Journal $\mathbf{1 4}$ $103-110$.

Wu H, Kanatous SB, Thurmond FA, Gallardo T, Isotani E, Bassel-Duby R \& Williams RS (2002) Regulation of mitochondrial biogenesis in skeletal muscle by CaMK. Science 296, 349-352.

Wu H, Naya FJ, McKinsey TA, Mercer B, Shelton JM, Chin ER, Simard AR, Michel RN, Bassel-Duby R, Olson EN \& Williams RS (2000) MEF2 responds to multiple calcium-regulated signals in the control of skeletal muscle fiber type. EMBO Journal 19, 1963-1973.

Zhu X, Hadhazy M, Wehling M, Tidball JG \& McNally EM (2000) Dominant negative myostatin produces hypertrophy without hyperplasia in muscle. FEBS Letters 474, 71-75. 
https://doi.org/10.1079/PNS2004362 Published online by Cambridge University Press 